\title{
Usefulness of the Modified Clip-with-Line Method for Endoscopic Mucosal Treatment Procedure
}

\author{
Koichi Soga, Takahito Shimomura, Toshio Suzuki, Tesshin Tei, Tomohiko Usui, Yoshikazu Inagaki, Kyoichi Kassai, \\ Kenji Itani
}

Nishijin Hospital, Department of Internal Medicine, Kyoto, Japan

\author{
Address for correspondence: \\ Koichi Soga \\ Department of Internal \\ Medicine, Nishijin Hospital \\ 1035 Mizomae-cho, \\ Kamigyo-ku, \\ Kyoto 602-8319, Japan \\ sogatti@koto.kpu-m.ac.jp
}

Received: 03.07.2018

Accepted: 05.08.2018

\begin{abstract}
Endoscopic submucosal dissection (ESD) might be difficult to perform in some cases even for experienced endoscopists. Recently, various traction methods have been introduced to facilitate ESD procedures, such as clip-with-line (CL), external forceps, clip and snare, internal traction, double scope, and magnetic anchor. The traction procedure using the CL method enhances the visibility of the operation field during ESD. The original CL method is performed as follows: the clip with the line (e.g., dental floss) is attached to the edge of the lesion. Traction can be produced by pulling the line gently, thus achieving a sufficient visualization of the submucosal layer to identify an accurate cutting line. Herein, we present a useful modified two-CL method by pulling the appropriate normal mucosa to enhance the visibility of the operation field during ESD procedure. Thus, the traction methods, including our modified CL technique, might reduce the procedure time and complications.
\end{abstract}

Key words: clip-with-line - endoscopic submucosal dissection - traction method.

Abbreviations: CL: clip-with-line; ESD: endoscopic submucosal dissection; LGE: lower gastrointestinal endoscopy; UGE: upper gastrointestinal endoscopy.

\section{INTRODUCTION}

Endoscopic submucosal dissection (ESD) has been used to treat neoplasms in the esophagus, duodenum, colon, and rectum. This technique can provide en bloc resection of gastrointestinal mucosal lesions regardless of size [1-4]. Despite these achievements, some ESD procedures are difficult to perform, even for experienced endoscopists. Recently, various traction methods have been introduced to facilitate ESD technique, such as clip-with-line (CL), external forceps, clip and snare, internal traction, double scope, and magnetic anchor [5]. The traction procedure using a clip-with-line (CL) method enhances the visibility of the operation field during ESD [6]. Several retrospective clinical case-control studies demonstrated that this method shortened the operation time, as well as ensured the safety of ESD $[7,8]$. The original CL method is as follows: the clip with the line (e.g., dental floss) is attached to the edge of the lesion. Traction can be produced by pulling the line gently, thus achieving sufficient visualization of the submucosal layer to identify an accurate cutting line. The following case reports present a useful modified two-CL method by pulling the appropriate normal mucosa to enhance the visibility of the operation field during ESD.

\section{CASE REPORTS}

\section{Case 1}

A 75-year-old woman underwent upper gastrointestinal endoscopy in our hospital, which evidenced a $10-\mathrm{mm}$ diameter polyp-shaped gastric cancer on the giant curvature of the antrum, to the pyloric ring (Fig.1A) with peristalsis forward to the duodenal bulb (Fig. 1B). The tumor was entirely visualized by traction with biopsy forceps on preview endoscopy (Fig. 1C). In the ESD procedure, a clip applicator device was inserted into the accessory channel of the endoscope, and a clip was mounted onto the tip of the clip device. Then, the dental floss was tied to one arm of the clip by a surgeon's knot. Before endoscopic intubation of the stomach, the clip was withdrawn inside the 

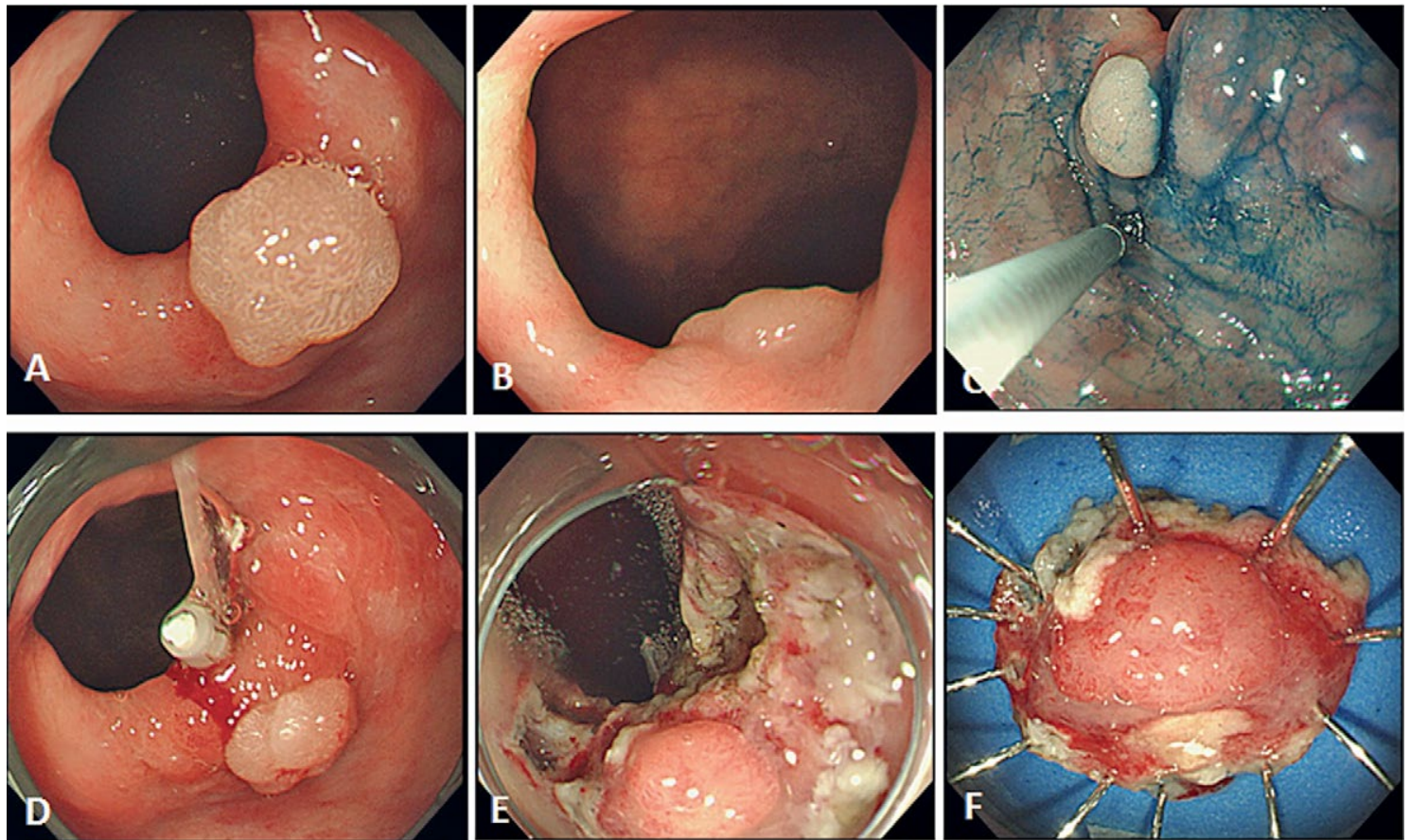

Fig. 1. The modified clip-with-line method on upper gastrointestinal endoscopy. Endoscopic appearance of a 10-mm diameter superficially elevated polyp-shaped gastric cancer on the giant curvature of the antrum to the pyloric ring (A) with peristalsis forward to the duodenal bulb (B). The tumor could be visualized entirely by traction with biopsy forceps on preview endoscopy (C). The clip was tied with a dental floss and attached over the distal edge of the tumor lesion, and the line was pulled gently in the oral direction (D). In D and E, we visualized the entire appearance of tumor, and submucosal dissection was completed without any complications (F).

channel to avoid trauma during endoscope insertion. When the scope reached the tumor lesion, the clip was tied with a dental floss attached over the distal edge of the tumor; the line was pulled gently in the oral direction by an assistant (Fig. 1D). The visibility of the operation field during ESD was thus enhanced. The submucosal dissection was completed without any trouble. Figures $1 \mathrm{E}$ and $1 \mathrm{~F}$ show the gross appearance of the tumor.

\section{Case 2}

A 71-year-old man underwent lower gastrointestinal endoscopy (LGE) in our hospital and was diagnosed with a 3-mm diameter, superficially elevated polyp-shaped colonic polyp into the appendix. On general appearance without any traction of the appendix, the polyp was recognized based on the appearance of the exposed part (Fig. 2A). The tumor could be visualized entirely by traction at the orifice with biopsy forceps on preview endoscopy. A clip applicator device was prepared outside the endoscope. A clip was mounted onto the tip of the clip device, the clip was opened by half, and the dental floss was then tied to one arm of the clip by a surgeon's knot. A small loop was then made on the other side of dental floss. After this preparation, the clip with the looped line was put back into the applicator. A clip applicator device was inserted into the accessory channel of the endoscope. When the clip device reached the lesion, the clip was tied to a dental floss and attached over the appendix orifice, out of the polyp lesion (Fig. 2B). Then, another clip was hooked in the looped line (Fig. 2C), and the appropriate length was adjusted by looping the line with rotating the clip (Fig. 2D). This second clip, with a looping dental floss was tied to the normal colonic mucosa.
The traction permitted a good visualization of the entire polyp, and we were able to perform cold polypectomy easily (Fig. 2E and $2 \mathrm{~F}$ ). After the polypectomy procedure, the looping line was put back to release the line traction (Fig. 2G).

\section{DISCUSSION}

Adequate tissue tension and a clear view of the tissue to be dissected are important for effective and safe ESD (Fig. 3) as well as various endoscopic procedures. Recently, new concepts have been developed to facilitate the ESD procedure, one of which is the traction method [9]. This manoeuver facilitates the visualization of the submucosal layer, thus enabling an accurate identification of the cutting line and submucosal vessels. Subsequently, this might reduce the procedure time and complications and lead to more widespread adoption of ESD. The feasibility and advantages of the CL method in gastric $[7,10]$ and esophageal ESD $[8]$ have been demonstrated by previous clinical case-control studies. The original CL method uses a clip, with a silk line tied on its arm, which is attached to the tip of the clip applicator device and inserted through the working channel. The endoscope, with the line along the outside of the scope, is inserted. Then, the clip with the line is attached to the edge of the lesion. By pulling the line gently, the tumor is tracked, thus achieving sufficient visualization of the submucosal layer to identify an accurate cutting line (Fig. 3).

In our patients, we performed endoscopic procedures more conveniently by applying the CL traction method. We emphasized that the original CL method was used to tract the tumor lesion, although we performed the modified CL 

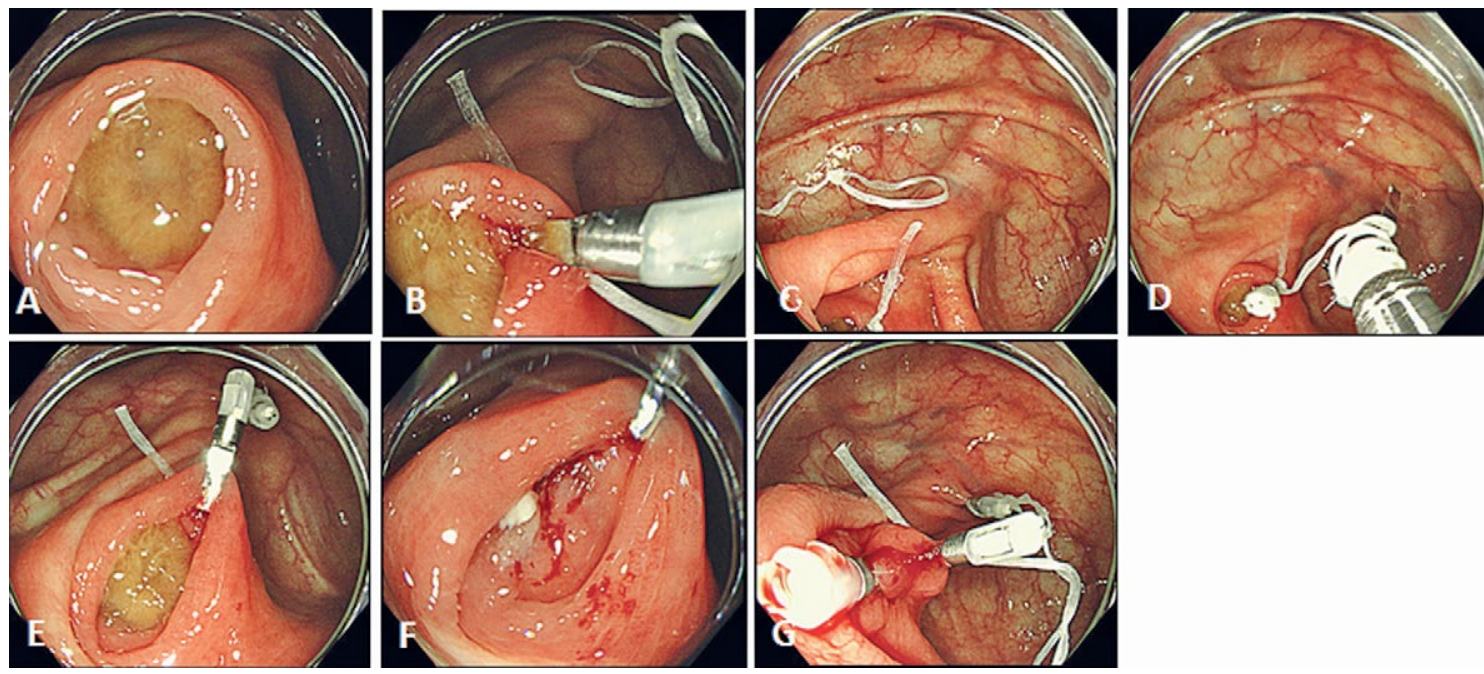

Fig. 2. The modified clip-with-line method on lower gastrointestinal endoscopy. Endoscopic appearance of a 3-mm diameter colonic polyp localized on the appendix. Without any traction of the appendix, the polyp is recognized only on the appearance of the exposed part (A). First clip was tied with a loop dental floss and attached over the appendix orifice out of the polyp lesion (B). A second clip was hook on the opposite side of the loop made-line (C). The proper length was adjusted by rotating the clip (D). The clip with a looping dental floss was tied to visualize the entire polyp, and we performed cold polypectomy procedure easily (E and F). After the polypectomy, the looping line was put back to release the line traction $(\mathrm{G})$.
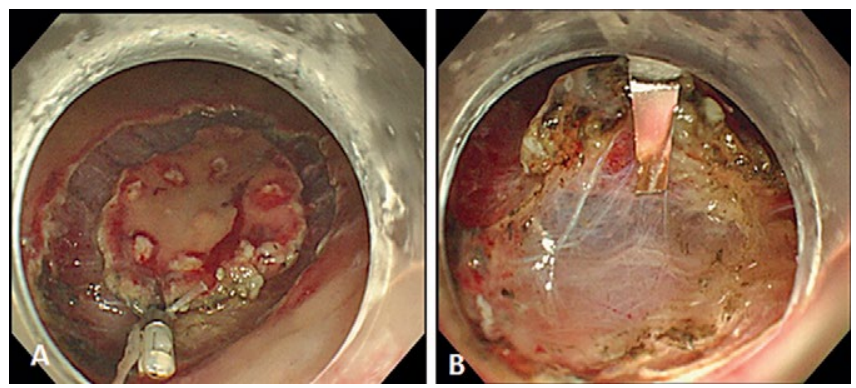

Fig. 3. Original clip-with-line method for gastric tumor The clip with line is attached to the edge of the lesion (A). Traction can be produced by pulling the line gently, thus achieving sufficient visualization of the submucosal layer to identify the accurate cutting line (B).

method, which tracts the normal mucosa. In Case 1, CL was attached over the distal side of the tumor lesion. The traction maneuver allowed a better visualization of the distal side of the tumor, so the cutting line for ESD was correctly identified. We often struggled to perform ESD for the tumor localized on the distal site of the pyloric ring as the working space is narrow and the approach from the distal duodenal site, is not easy. In Case 2, the first clip was tied with a loop (made of dental floss) attached over the appendix orifice out of the lesion. A second clip was hooked into the loop; the length of the line was adjusted by rotating the clip. Dissecting the cecum in proximity to the appendicle orifice might be difficult due to endoscopic instability and special anatomical situation of the appendix often. In the modified CL, the dental floss was tied and attached in the appropriate position to visualize the entire tumor and perform the polypectomy easily. In Case 2 , using a loop, we were able to control the length of the line and the traction force. Applying the CL method in various situations, the endoscopic procedure might be performed easily. The instrument preparation is easy. When we finished the endoscopic procedure, the line was easily cut. In Case 2 , the mucosal tension disappeared after rolling off the looped line around the clip. We consider that the CL method is necessary to simulate clip placement, force, and directions by traction. In our patients, the tumor could be visualized entirely by traction with biopsy forceps on preview endoscopy.

\section{CONCLUSION}

The modified CL method was effective for evaluating and resecting the lesions that are difficult to recognize. The original traction technique using the CL method was essentially used to facilitate the visualization of the submucosal layer, thus enabling accurate identification of the cutting line and submucosal vessels. Traction methods, including our modified method, represent a promising approach to help reduce the procedure time and complications and may lead to a more widespread adoption of endoscopic procedure.

\section{Conflicts of interest: None to declare.}

Authors' contributions: K.S. was responsible for devising the method and writing the manuscript. All the other authors participated equally in the work.

\section{REFERENCES}

1. Hosokawa K, Yoshida S. Recent advances in endoscopic mucosal resection for early gastric cancer. Gan To Kagaku Ryoho 1998;25:476483.

2. Ono H, Kondo H, Gotoda T, et al. Endoscopic mucosal resection for treatment of early gastric cancer. Gut 2001;48:225-229. doi:10.1136/ gut.48.2.225

3. Nakajima T, Saito Y, Tanaka S, et al. Current status of endoscopic resection strategy for large, early colorectal neoplasia in Japan. Surg Endosc 2013;27:3262-3270. doi:10.1007/s00464-013-2903-X 
4. Saito Y, Uraoka T, Yamaguchi Y, et al. A prospective, multicenter study of 1111 colorectal endoscopic submucosal dissections (with video). Gastrointest Endosc 2010;72:1217-1225. doi:10.1016/j. gie.2010.08.004

5. Tsuji K, Yoshida N, Nakanishi H, Takemura K, Yamada S, Doyama H. Recent traction methods for endoscopic submucosal dissection. World J Gastroenterol 2016;22:5917-5926. doi:10.3748/wjg.v22.i26.5917

6. Oyama T, Kikuchi Y, Shimaya S. Endoscopic mucosal resection using a hooking knife (hooking EMR). Stomach Intest 2002;37:1155-1161.

7. Suzuki S, Gotoda T, Kobayashi Y, et al. Usefulness of a traction method using dental floss and a hemoclip for gastric endoscopic submucosal dissection: a propensity score matching analysis (with videos). Gastrointest Endosc 2016;83:337-346. doi:10.1016/j. gie.2015.07.014

8. Ota M, Nakamura T, Hayashi K, et al. Usefulness of clip traction in the early phase of esophageal endoscopic submucosal dissection. Dig Endosc 2012;24:315-318. doi:10.1111/j.1443-1661.2012.01286.x

9. Imaeda $\mathrm{H}$, Hosoe $\mathrm{N}$, Kashiwagi $\mathrm{K}$, et al. Advanced endoscopic submucosal dissection with traction. World J Gastrointest Endosc 2014;6:286-295. doi:10.4253/wjge.v6.i7.286

10. Jeon WJ, You IY, Chae HB, Park SM, Youn SJ. A new technique for gastric endoscopic submucosal dissection: peroral traction-assisted endoscopic submucosal dissection. Gastrointest Endosc 2009;69:29-33. doi:10.1016/j.gie.2008.03.1126 